\title{
O uso da técnica de "Linkage" de sistemas de informação em estudos de coorte sobre mortalidade neonatal*
}

\section{The use of the 'Linkage' of information systems in cohort studies of neonatal mortality}

\author{
Marcia Furquim de Almeida e Maria Helena P. de Mello Jorge \\ Departamento de Epidemiologia da Faculdade de Saúde Pública da Universidade de São Paulo. São \\ Paulo, SP - Brasil
}

\begin{abstract}
Resumo
Discute-se o uso da "linkage"dos Sistemas Oficiais de Informação de Nascido Vivo (SINASC) e de Óbitos (SIM) em estudos de mortalidade neonatal. Essa técnica baseia-se na "ligação" dos bancos de dados obtidos a partir das informações existentes nesses sistemas, o que possibilita o emprego de estudos do tipo de coorte. O estudo foi realizado no Município de Santo André, Região Metropolitana de São Paulo, Brasil. São apresentados os cuidados metodológicos que foram empregados para evitar a presença de viéses de seleção e de efeito, que podem ocorrer. O uso da "linkage" mostrou-se operacionalmente viável, permitindo obter as probabilidades de morte e os riscos relativos dos nascidos vivos, expostos e não expostos, às variáveis que são objeto de registro na declaração de nascido vivo, identificando-se, desta maneira, os recém-nascidos de risco. Essa técnica, de baixo custo operacional, visto que utiliza dados já registrados, permite um dimensionamento mais adequado da assistência pré-natal e ao parto.
\end{abstract}

Sistemas de informação. Fatores de risco. Mortalidade neonatal.

\begin{abstract}
The utilization of record linkage of the mortality and birth information systems in studies of neonatal mortality is presented. The record linkage was used to obtain a cohort of live births and neonatal deaths in Santo André county, located within greater S. Paulo, in 1992. The procedures applied in order to avoid selection and effect biases, are discussed. The use of linked data allows the probabilities of neonatal deaths according to the exposure status of the variables which are registered on the birth certificate, and the identification of the live born at risk, to be calculated. Another advantage of the record linkage is the low financial cost of this type of study, because it uses information already registered.
\end{abstract}

Information systems. Risk factors. Neonatal mortality.

\footnotetext{
* Extraído da Tese de Doutorado "Mortalidade neonatal em Santo André" apresentada à Faculdade de Saúde Pública da USP em 1995. Subprojeto da Pesquisa SINASC (CNPq/OSP/OMS Proc. 501590/91-9).

Correspondência para / Correspondence to: Marcia Furquim de Almeida - Av. Dr. Arnaldo, 715 - 01246-904 São Paulo, SP - Brasil. Fax: (011) 282.2920

Edição subvencionada pela FAPESP. Processo 95/2290-6.

Recebido em 17.7.1995. Aprovado em 14.11.1995.
} 


\section{INTRODUÇÃO}

A implantação do Sistema de Informação sobre Nascidos Vivos (SINASC) permitiu a obtenção de dados mais detalhados e fidedignos sobre nascimentos vivos do que os existentes anteriormente (Mello Jorge e col. $\left.{ }^{9}, 1993\right)$. A Declaração de Nascido Vivo (DN), documento básico de coleta de dados do SINASC, tornou viável obter informações populacionais sobre peso ao nascer, duração da gestação, tipo de parto e hospital de nascimento, que se constituem em elementos importantes na análise da mortalidade infantil.

Ao lado desses ganhos, há a possibilidade de realização de estudos de mortalidade infantil que utilizem a "linkage" dos sistemas de informação de nascimentos e óbitos, a exemplo do que já ocorre em outros países, como os Estados Unidos (CDC $\left.{ }^{2}, 1989\right)$ e Noruega (Lie e col. $\left.{ }^{6}, 1987\right)$. O emprego dessa técnica permite uma maior utilização dos dados existentes nos diferentes sistemas de informação, com estudos de relativo baixo custo operacional.

Nos Estados Unidos, a realização do projeto "National Infant Mortality Surveillance" (NIMS), que tinha por objetivo a análise comparativa de dados obtidos por meio da "linkage"dos sistemas de informação, de nascimentos e óbitos, com base nas coortes de nascidos vivos de 1960 e 1980, mostrou que esta técnica possibilitava um detalhamento maior do comportamento da mortalidade infantil nesse período (Buehler e col. ${ }^{1}$, 1987; Prager e col. ${ }^{11}$, 1987; Sappenfield e col. ${ }^{12}$, 1987; CDC $^{2}$, 1989). A partir da avaliação dos resultados desse projeto, o "National Center of Health Statistics" (NCHS) iniciou a divulgação de dados nacionais de mortalidade infantil, com base nas coortes anuais de nascidos vivos, utilizando a técnica de "linkage" dos bancos de dados de nascimentos e óbitos infantis (CDC $\left.{ }^{3}, 1994\right)$.

A "linkage" de bancos de dados vem sendo utilizada com diferentes finalidades. Mac Mahon e Pugh ${ }^{7}$, 1970 mencionam o seu uso em estudos de morbidade em serviços de saúde de diferentes níveis de complexidade. Esta técnica vem sendo empregada no Canadá, na identificação de recém-nascidos de risco para anomalias congênitas (Trimble ${ }^{13}$, 1975). Leibson e col. ${ }^{5}, 1992$ descrevem o seu uso em estudos longitudinais envolvendo a ligação de bancos de dados de diferentes serviços de saúde e de mortalidade, na cidade de Rochester (EUA). Este estudo evidenciou a ocorrência de mudança na definição de diagnósticos, ao longo do tempo, devido à incorporação de tecnologia, tornando-os mais precisos e possibilitando, dessa forma, a identificação de novos agravos.
A tradução literal de "linkage" é "ligação", consistindo na ligação ou unificação de dois ou mais bancos de dados. O emprego da "linkage" pressupõe a existência de informações registradas em documentos padronizados e individualizados, de modo a permitir a identificação do mesmo indivíduo nos dois ou mais bancos. Nos estudos de mortalidade infantil, parte-se de uma coorte de nascidos vivos e pareiam-se as Declarações de Óbito (DO) relativas aos óbitos infantis com as respectivas DN.

Contudo, a realização desse tipo de estudo requer o emprego de procedimentos que visem a evitar a presença de viéses de seleção e de efeito. Para a definição da coorte de nascidos vivos é necessário identificar possíveis duplicações de emissão de DN, de acordo com as fontes de obtenção das mesmas, pois a existência de duplicação irá resultar na superestimação dos expostos à ocorrência do efeito. Laurenti e col. ${ }^{4}, 1985$, descrevem a possibilidade de erros de classificação para nascidos vivos que morrem logo após o nascimento, os quais podem ser indevidamente classificados como nascidos mortos. A presença deste erro iria subestimar o número de nascidos vivos expostos ao risco de morte neonatal, e, de outro lado, iria subestimar, também, a ocorrência de óbitos neonatais na coorte considerada.

O objetivo do presente trabalho é verificar a possibilidade de utilizar a "linkage" dos sistemas oficiais de informação de mortalidade (SIM/MS) e de nascidos vivos (SINASC/MS) para o estudo da mortalidade neonatal no Município de Santo André, Região Metropolitana de São Paulo.

A escolha de Santo André como área de estudo teve várias razões. Em primeiro lugar, por ser um dos munícipios em que foi realizada a pesquisa "Avaliação do Sistema de Informações de Nascimentos e o Uso de seus Dados em Epidemiologia e Estatísticas de Saúde" (Mello Jorge e col. ${ }^{9}, 1993$ ), que tinha, entre outras finalidades, avaliar o grau de cobertura do SINASC e a qualidade das informações registradas na DN. Em segundo lugar, é uma área com 100\% de urbanização e, portanto, o número de partos domiciliares deve ser reduzido. Em terceiro, o Munícipio faz parte da Região Metropolitana, onde se encontra mais de $50 \%$ da população do Estado de São Paulo e, por último, a proximidade da Capital do Estado representava menores custos operacionais.

\section{MATERIAL E MÉTODO}

Considerou-se como população de estudo o conjunto de nascidos vivos ocorridos no Município de Santo André, no período de 1/1/92 a 30/6/92, filhos de mulheres residentes no munícipio. Faz parte desta coorte, também, os 
óbitos com menos de 28 dias de vida ${ }^{8}$, originados destes nascidos vivos, compreendendo as mortes ocorridas entre 1/1/92 a 27/7/92, em Santo André ou em outros munícipios.

Foram utilizadas as DN e as DO para a obtenção de informações desses eventos.

As DN foram coletadas no Escritório Regional de Saúde (ERSA-9), da Secretaria de Estado da Saúde de São Paulo,que cobre a região do Município de Santo André. De acordo com o fluxo do SINASC, no Estado de São Paulo, o ERSA recebe as DN preenchidas pelos hospitais do munícipio (DN-HOSP) e as DN relativas aos nascimentos domiciliares que são preenchidas pelos Cartórios de Registro Cívil (DN-CART). Foram obtidas 6.563 DNHOSP e 59 DN-CART.

Tendo em vista a existência de algumas falhas de cobertura do SINASC, para esse munícipio, foram utilizadas as DN preenchidas pela pesquisa-base (DN-PESQ), já mencionada (Mello Jorge e col. $\left.{ }^{9}, 1993\right)$. Este conjunto de DN referia-se a nascimentos hospitalares ocorridos em Santo André durante o período de referência do estudo, para os quais os hospitais não haviam emitido as respectivas DN. Foram obtidas 42 DN-PESQ.

No total, foram coletadas 6.664 DN correspondentes a nascimentos hospitalares e domiciliares ocorridos em Santo André, no período de referência.

As declarações de óbito neonatal foram coletadas nos Cartórios de Registro Cívil para os óbitos ocorridos em Santo André (55 DO ) e na Fundação Sistema Estadual de Análise de Dados (SEADE), para aqueles ocorridos em outros munícipios (40 DO).

Foram coletadas, ainda, nos Cartórios de Registro Civil, 51 DO-Fetais relativas aos óbitos fetais de mães residentes, ocorridos em Santo André, no período de 1/1/ 1992 a 30/6/1992, para posterior verificação dos eventos.

Como fonte complementar de dados foram utilizados os prontuários médicos, quer por meio de consulta direta, quer por meio das informações obtidas pela pesquisa-base ${ }^{9}$. A consulta aos prontuários tinha por objetivo verificar possíveis falhas de cobertura dos sistemas de informação e a existência de erros de classificação dos eventos estudados.

O programa Epi Info - Versão 5.0 foi utilizado para os procedimentos de verificação dos documentos e para a "linkage" dos bancos de dados, sendo que a conferência de cada caso foi realizada manualmente.

\section{DEFINIÇÃO DA COORTE DE NASCIDOS VIVOS, IDENTIFICAÇÃO DOS ÓBITOS NEONATAIS E "LINKAGE"}

\section{- Verificação do Município de Residência nas DN}

O Município de Santo André é uma área de invasão de nascimentos; portanto, fazia-se necessário identificar o munícipio de residência da mãe. Este procedimento foi realizado em duas etapas. Inicialmente comparou-se o munícipio de residência regis- trado no conjunto 6.664 DN com aquele obtido nos prontuários médicos pela pesquisa-base ${ }^{9}$. Nos casos em que havia divergência de registros, o endereço era localizado por meio de consulta ao "Guia de São Paulo - 1993".

Com esse procedimento, obteve-se o número de nascimentos vivos ocorridos e de mães residentes em Santo André, sendo que faziam parte desse conjunto 3.155 DN-HOSP; 55 DN-CART e 26 DN-PESQ, Totalizaram-se, nessa etapa, $3.236 \mathrm{DN}$, sendo as restantes excluídas do estudo (Fig. 1).

\section{- Verificação de Duplicação de Emissão da DN}

Inicialmente foram utilizadas as variáveis data de nascimento e sexo e, na fase seguinte, a variável nome da mãe para identificar a existência de duplicação de DN entre as fontes de obtenção de dados utilizadas.

Verificou-se que havia duplicação para aquelas DN preenchidas pelos cartórios e hospitais, sendo excluídas 5 DN-CART ( referiam-se a nascimentos hospitalares que os cartórios haviam, indevidamente, considerado como domiciliares). Dessa forma, permaneceram em estudo 50 DN-CART (Fig. 1).

Observou-se que existiam duplicações entre as DN obtidas pela pesquisa-base e as emitidas pelos cartórios (3 nascimentos possuiam DN-PESQ e DNCART), sendo excluídas as DN-PESQ, pois, ainda que incorretamente, o sistema oficial de informações conseguiu captar esses eventos. Portanto, passaram a fazer parte do estudo 23 DN-PESQ (Fig. 1).

Não é esperado que sejam preenchidas DN para nascidos mortos. Porém, devido à compreensão incorreta das instruções para o preenchimento das DN (Ministério da Saúde ${ }^{10}, 1989$ ), este erro pode ocorrer. Para esta verificação, partiu-se das variáveis data de nascimento, sexo e hospital de nascimento registradas nas 51 DO-Fetais. Selecionou-se o conjunto de DN-HOSP que apresentavam estas mesmas informações. Utilizando-se a variável nome da mãe, compararam-se as DO-Fetais, caso a caso, com o conjunto de DN-HOSP já selecionado. Verificou-se que os hospitais haviam emitido $3 \mathrm{DN}$ para óbitos fetais. Estas DN foram excluídas da coorte, permanecendo 3.152 DN-HOSP no estudo (Fig. 1).

\section{- Definição da Coorte de Nascidos Vivos}

Após esse conjunto de procedimentos, a coorte passou a ser formada por 3.152 DN-HOSP, $50 \mathrm{DN}$ CART e 23 DN-PESQ, totalizando 3.225 DN referentes a nascidos vivos de mães residentes e ocorridos em Santo André (Fig. 1). 


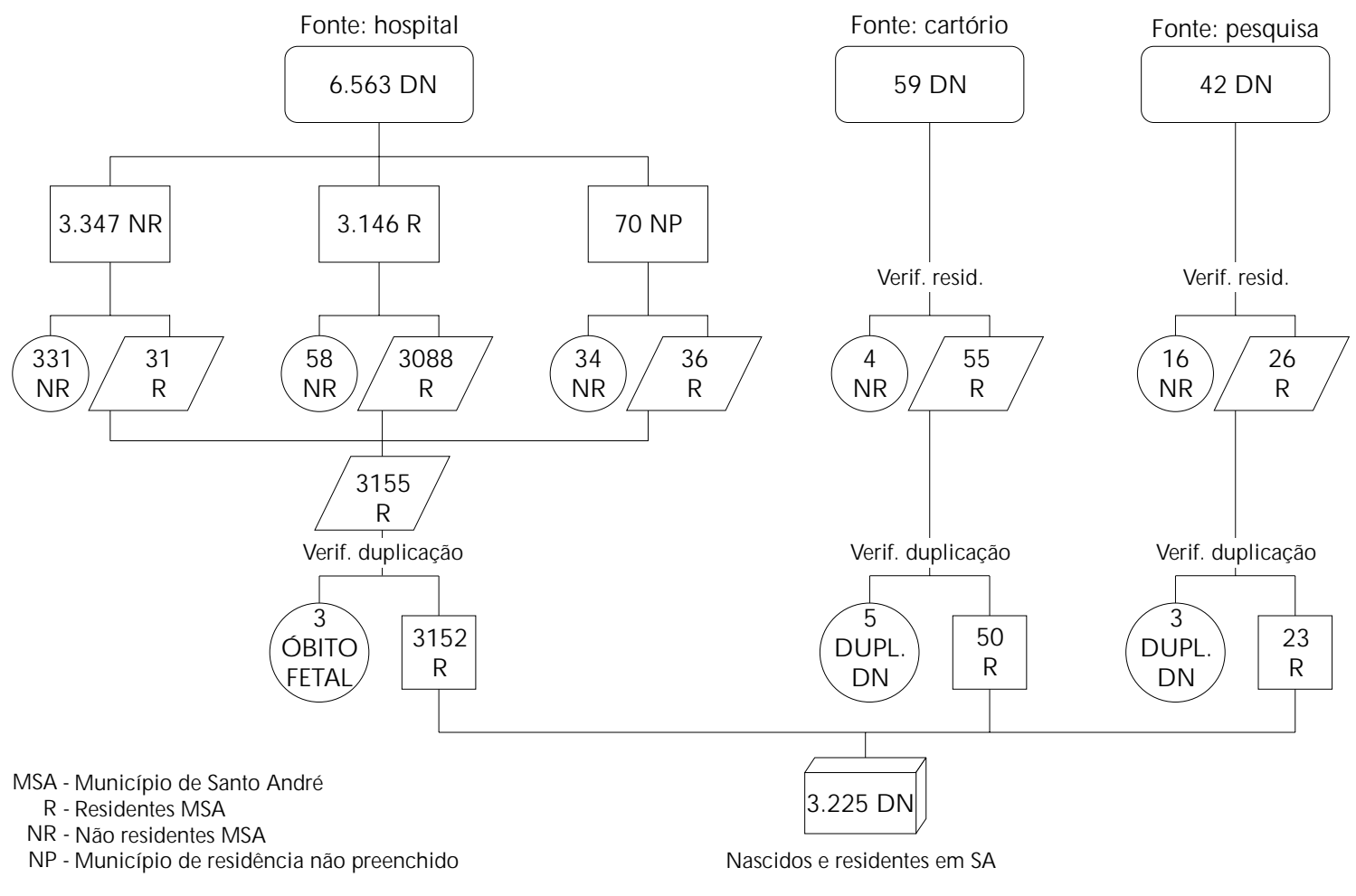

Figura 1 - Coorte de nascidos vivos, segundo fonte de obtenção das Declarações de $\mathrm{N}$ ascido Vivo e procedimentos para sua identificação.

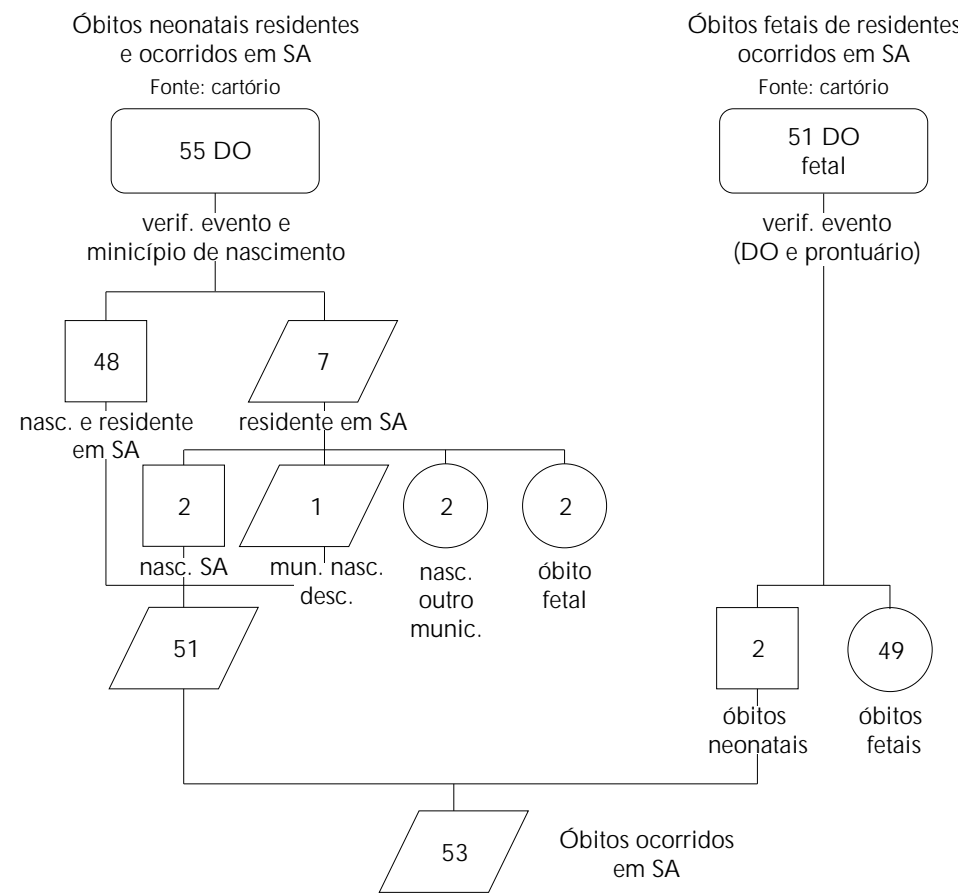

Ó bitos neonataisde residentes ocorridos em outros municípios
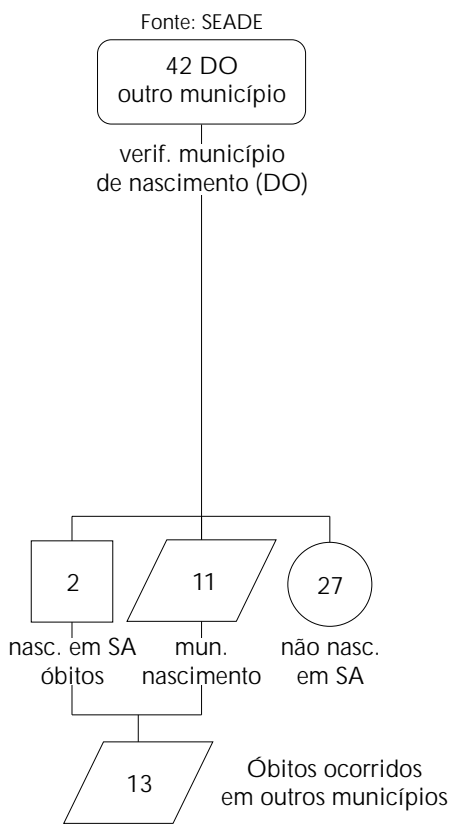

Figura 2 - Ó bitos neonatais, segundo fonte de obtenção das Declarações de Ó bito e procedimentos para sua identificação. 


\section{• Óbitos Ocorridos em Santo André}

Para identificar os óbitos neonatais pertencentes à coorte em estudo, fazia-se necessário identificar o município de nascimento, bem como verificar a existência de possíveis erros de classificação, que resultariam no registro de óbitos neonatais como nascidos mortos.

Foram obtidas 55 DO neonatais de residentes em Santo André. Deste conjunto, constatou-se que em 50 DO os nascimentos também haviam ocorrido neste município, o que indicava que esses óbitos, possivelmente, pertenciam à coorte de nascidos vivos considerada.

Não foi possível identificar o município de nascimento em 5 DO devido a falhas de preenchimento. Contudo, estas DO foram mantidas em estudo com o objetivo de verificar esta informação em outra fonte (Fig. 2).

Observou-se, ainda, que 2 DO apresentavam falhas de preenchimento no campo 1 (identificação de óbito fetal) e campo 5 (idade ao morrer). Dessa maneira, não se tinha certeza se esses óbitos eram neonatais ou fetais. Essas DO, também, foram mantidas no estudo para posterior verificação (Fig. 2).

\section{- Verificação das Informações dos Óbitos nos Prontuários Hospitalares}

Tendo em vista a possibilidade de DO-Fetais terem sido preenchidas para óbitos neonatais, as informações das 51 DO-Fetais de Santo André foram verificadas junto aos prontuários médicos. Observouse que este tipo de erro estava presente em 2 óbitos: o primeiro recém-nascido havia falecido aos $10 \mathrm{~min}$ de vida e o segundo aos $45 \mathrm{~min}$. Procedimento semelhante foi adotado para o conjunto de 55 óbitos neonatais ocorridos em Santo André. Verificou-se que as 2 DO que apresentavam os campos 1 e 5 (identificação de óbito fetal e idade ao morrer) não preenchidos correspondiam a óbitos fetais, sendo portanto excluídas do estudo (Fig. 2).

Com relação às $5 \mathrm{DO}$ em que não havia sido possível identificar o município de nascimento, a consulta aos prontuários médicos revelou que duas dessas crianças haviam nascido em Santo André, sendo incluídas na pesquisa. Em um dos óbitos deste conjunto não foi possível verificar o munícipio de nascimento, quer com a consulta aos prontuários quer com as informações disponíveis no Serviço de Verificação de Óbitos. Contudo, esta DO foi mantida no estudo, mesmo não se tendo a certeza que este óbito pertencia à coorte de nascidos vivos considerada.
Duas outras DO foram excluídas, pois referiam-se a crianças nascidas em outros munícipios. Dessa maneira, totalizaram-se 53 DO relativas a óbitos neonatais, ocorridos no Munícipio de Santo André, passíveis de pertencer à coorte estudada (Fig. 2).

\section{- Óbitos Ocorridos em Outros Municípios}

Obteve-se na Fundação SEADE 40 DO neonatais de residentes no Município de Santo André. Para identificar se esses óbitos pertenciam à coorte, verificou-se o município de nascimento (campo 17). Observou-se que em 27 óbitos, o município de nascimento registrado correspondia a outras áreas, sendo que essas DO foram excluídas (Fig. 2).

Das 13 DO restantes, em apenas 2 casos o município de nascimento era Santo André. Nas outras 11, o campo 17 não estava preenchido ou havia sido preenchido incorretamente, não tendo sido possível identificar se esses óbitos pertenciam ou não a crianças nascidas nesse munícipio, razão pela qual essas DO foram mantidas no estudo.

\section{- "Linkage"}

Obteve-se um total de 66 DO, passíveis de pertencer à coorte de nascimentos vivos considerados (53 correspondiam a óbitos neonatais ocorridos no Município de Santo André e 13 a óbitos ocorridos em outros municípios). Estas DO foram, então, pareadas com as 3.225 DN relativas aos nascidos vivos da coorte $\left(\mathrm{CDC}^{2}, 1989\right)$. Para realizar o pareamento foram utilizadas as variáveis data de nascimento e sexo registradas nas DO para selecionar o conjunto de DN que apresentavam a mesma data de nascimento e sexo. Neste conjunto, a identificação da DO correspondente à respectiva DN foi feita manualmente, caso a caso, utilizando-se a variável nome da mãe.

Com este procedimento foi possível parear 55 DO com as respectivas DN (Fig. 3). Foram excluídas 11 DO, sendo que 1 destes óbitos havia ocorrido em Santo André e os outros 10 em outros municípios. Estes casos pertenciam ao conjunto de DO que haviam sido mantidas em estudo, mesmo não se conhecendo o município de nascimento dessas crianças, conforme citado anteriormente (Fig. 3).

As $55 \mathrm{DO} / \mathrm{DN}$ pareadas representam os óbitos neonatais e as 3.170 DN não pareadas representam os sobreviventes da coorte de 3.225 nascidos vivos (Fig. 3). Desta maneira, obteve-se uma coorte retrospectiva e estática, dado que os eventos já haviam ocorrido e que foi fixado um período determinado de 


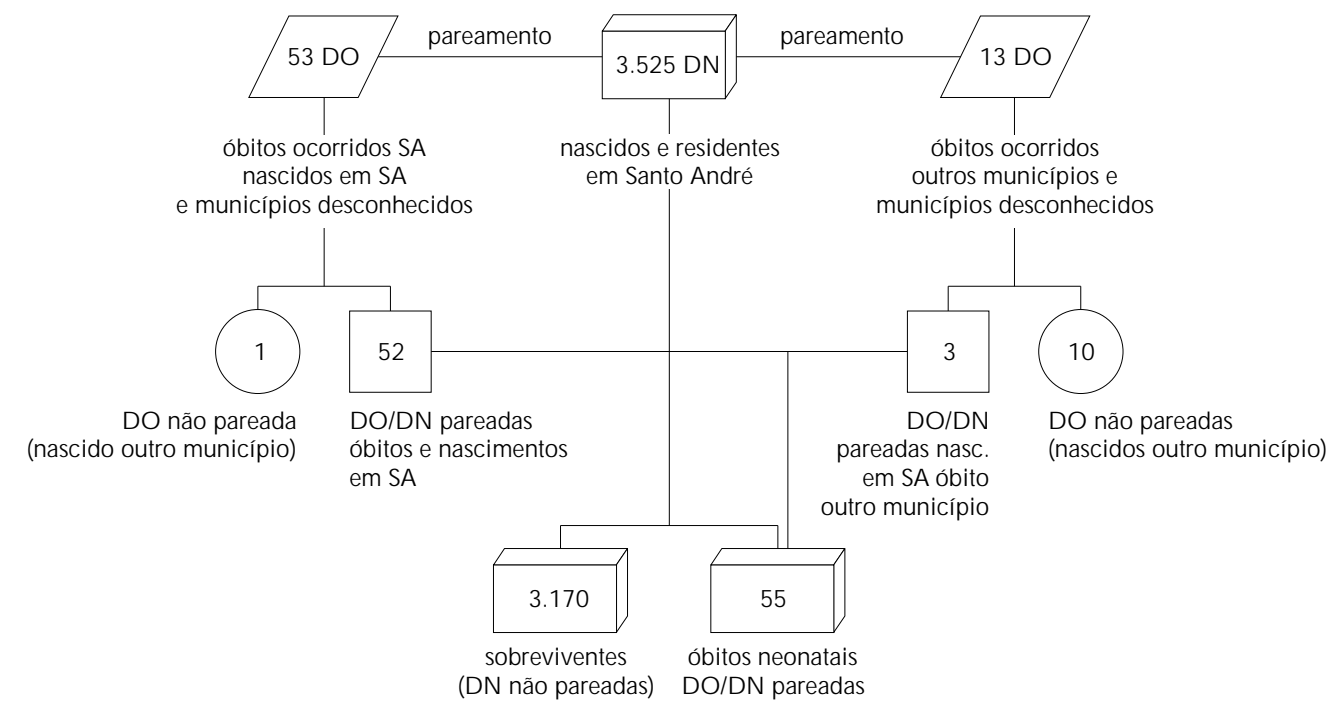

Figura 3 - Identificação dos óbitos neonatais da coorte de nascidos vivos ocorridos e de mães residentes em Santo André, utilizando a técnica "Linkage".

tempo de observação para a verificação do efeito, tendo-se como pressuposto a inexistência de migração e perdas de observação durante o período referido.

Após o pareamento, os bancos de dados originais (relativos às DN e DO) foram unificados, criando-se uma nova variável dicotômica relativa a óbitos e sobreviventes. Foram incorporadas ao banco de dados das DN apenas as variáveis que caracterizaram os óbitos (data e local do óbito, tipo de hospital e causas de morte). As demais variáveis da DO não foram consideradas, pois muitas já são objeto de registro na $\mathrm{DN}$, sendo que esta é a fonte comum para óbitos e sobreviventes.

\section{COMENTÁRIOS SOBRE A VIABILIDADE DA TÉCNICA}

Os resultados obtidos mostram que é possível utilizar a técnica de "linkage". Entretanto, analisando-se os dados da Tabela, que contém a distribuição dos óbitos neonatais e sobreviventes, segundo a fonte de obtenção das DN, verifica-se que a proporção de DN-PESQ entre os óbitos foi de 9\%, enquanto que nos sobreviventes correspondeu a $0,6 \%$, o que indica que a falha de emissão de DN nos hospitais foi maior para as crianças que morreram, possivelmente, concentrando-se naquelas com menor viabilidade.

Esses resultados mostram que há, por parte do serviços de saúde, uma compreensão incorreta das instruções existentes no "Manual de Preenchimento da DN" (Ministério da Saúde ${ }^{10}, 1989$ ), gerando esse tipo de falha de cobertura do sistema. Os Cartórios de Registro Civil também contribuem para a existência de falhas na cobertura do SINASC. Estes deveriam proceder, em primeiro lugar, ao registro do nascimento vivo para, posteriormente, registrar o óbito neonatal. Assim, deveriam exigir a apresentação da respectiva DN ou de prova de registro anterior de nascimento para, a seguir, providenciar o registro do óbito.

Tabela - Número de nascidos vivos, óbitos neonatais e sobreviventes segundo fonte de obtenção das DN

\begin{tabular}{|c|c|c|c|c|c|c|}
\hline \multirow{2}{*}{ Fonte } & \multicolumn{2}{|c|}{$\begin{array}{c}\text { Ó bitos } \\
\text { neonatais }\end{array}$} & \multicolumn{2}{|c|}{ Sobreviventes } & \multicolumn{2}{|c|}{ Total } \\
\hline & $\mathrm{N} N$ & $\%$ & $\mathrm{~N} N$ & $\%$ & $\mathrm{~N} N$ & $\%$ \\
\hline DN-Hosp & 50 & 90,9 & 3.102 & 97,8 & 3.152 & 97,7 \\
\hline DN-Cart & - & - & 50 & 1,6 & 50 & 1,6 \\
\hline DN-Pesq & 5 & 9,1 & 18 & 0,6 & 23 & 0,7 \\
\hline$\overline{\text { Total }}$ & 55 & 100,0 & 3.170 & 100,0 & 3.225 & 100,0 \\
\hline
\end{tabular}

Esse tipo de falha de cobertura do SINASC irá resultar na exclusão de óbitos neonatais da coorte de nascidos vivos, pois as DO referentes aos óbitos neonatais, provenientes de nascimentos para os quais não foi emitida DN, não serão passíveis de pareamento, levando a se subestimar a mortalidade na coorte em estudo.

A técnica de "linkage" dos sistemas oficiais de informação de nascimentos e mortalidade mostrouse viável para a realização de estudos de coorte em mortalidade neonatal. No entanto, em trabalhos que 
venham a utilizá-la, e que não possuam informações anteriores sobre o grau de cobertura do SINASC, considera-se importante o emprego de cuidados metodológicos que possam evitar a presença de possíveis viéses.

Como essa técnica baseia-se em informações individualizadas, é possível identificar os nascidos vivos expostos e não expostos às variáveis registradas na DN, obtendo-se a probabilidade de morte segundo a categoria de exposição a estas variáveis e, conseqüentemente, o risco relativo dos expostos em relação aos não expostos a estas características.

\section{REFERÊNCIAS BIBLIOGRÁFICAS}

1. BUEHLER, J.N. et al. Birth weigth specific infant mortality, 1960 and 1980. Public Health Rep., 102:151-61,1987.

2. CENTER FOR DISEASE CONTROL. National infant mortality surveillance, 1980. Atlanta, 1989. (NIMS Project).

3. CENTER FOR DISEASE CONTROL. Infant mortality by birthweight and other characteristics: United States, 1985 birth cohort. Vital Health Statist., Series 20 (24), 1994.

4. LAURENTI, R. et al. Estatísticas de saúde. São Paulo, EPU/EDUSP, 1985.

5. LEIBSON, C.L. et al. The compression of morbidity hypothesis: promise on pitfalls of using record linked data bases to acess secular trends in morbidity and mortality. Milbank Quart., 70: 127-55, 1992.

6. LIE, R.T. et al. Secular changes in early neonatal mortality in Norway, 1967-1981. Am. J. Epidemiol., 125: 1066-78, 1987.

7. MAC MAHON, B. \& PUGH, T.F. Epidemiology principles and methods. Boston, Little Brown Co., 1970. p. 95-8.
A "linkage" mostrou-se uma técnica de fácil emprego que possibilita a identificação dos recém-nascidos de risco e a sua mensuração em nível municipal e distrital. Com base nesses dados, é possível avaliar, de forma mais objetiva, a assistência pré-natal e ao parto, permitindo, também, o dimensionamento mais adequado dos cuidados necessários a serem dispensados aos recém-nascidos de risco. Esses procedimentos poderão contribuir para a redução da mortalidade neonatal, que se constitui, hoje, no componente predominante da mortalidade infantil do Estado de São Paulo.
8. MANUAL de classificação estatística internacional de doenças, lesões e causas de óbitos: $9^{a}$ Revisão, 1975. São Paulo, Centro Brasileiro de Classificação de Doenças da Organização Mundial da Saúde, 1985.

9. MELLO JORGE, M.H.P. et. al. Avaliação do sistema de informação sobre nascidos vivos e o uso de seus dados em epidemiologia e estatísticas de saúde. Rev. Saúde Pública, 27 (supl.), 1993.

10. MINISTÉRIO DA SAÚDE. Manual de instruções para o preenchimento da declaração de nascido vivo. Brasília, 1989.

11. PRAGER,K. et al. The NCHS pilot project to link birth and infant death records: stage 1. Public Health Rep., 102: 215 23,1987.

12. SAPPENFIELD, W.M. et al. Differences in neonatal and posneonatal mortality by race, birth weigth and gestational age. Public Health Rep., 102: 182-91,1987.

13. TRIMBLE, B.K. Record linkage and genetic registers. In Emery, A.E.H.\& Miller, J. Registers for detection and prevention of genetic diseases. New York, Stratton Co, 1975. p. $75-103$. 